\title{
AMPLITUDES OF OSCILLATIONS GOVERNED BY A MODIFIED VAN DER POL EQUATION ${ }^{1}$
}

\author{
BY \\ K. KLOTTER ${ }^{2}$ AND E. KREYSZIG ${ }^{3}$
}

1. Introduction; systems treated. The classical example of a system exhibiting self-sustained oscillations is the one described by the differential equation

$$
q^{\bullet}-\delta q^{\cdot}\left(1-\alpha^{2} q^{2}\right)+\kappa^{2} q=0,
$$

known as the "van der Pol differential equation". This differential equation cannot be solved in closed form. Properties of its solutions have been studied extensively, however, by various means and from different points of view. Sparked by a paper by Levinson and Smith [1], dealing with

$$
q^{\cdot}+g\left(q, q^{*}\right) q^{\cdot}+f(q)=0,
$$

a theory for this rather general class of differential equations was developed [2]. Because the assumptions made in that theory in regard to the coefficients of the differential equation are rather weak (in order to keep the conclusions general), no very specific results can be expected from it. Hence it seems desirable to investigate special differential equations or classes of differential equations, describing self sustained oscillations, which can be treated more fully; preferably differential equations for which closed form solutions, at least for the first integral, can be provided, if only in form of quadratures.

One of the authors [3] has drawn attention to two such differential equations, which may be called "modified" or "associated" van der Pol equations. They are

$$
q^{*}-\left(\operatorname{sgn} q^{\cdot}\right)(\epsilon / 2) q^{2}\left(1-\alpha^{2} q^{2}\right)+\kappa^{2} f(q)=0
$$

and

$$
q^{\cdot \cdot}-\left(\operatorname{sgn} q^{\cdot}\right)\left(\kappa^{2} s\right)\left(1-\beta^{2} q^{\cdot 2}\right)+\kappa^{2} f(q)=0 \quad(s>0) .
$$

Equation (3) [describing a first special class of self sustained oscillations] has been treated by the authors in a recent paper [4], and a more general form of that equation,

$$
q^{\cdot \cdot}-\left(\operatorname{sgn} q^{\cdot}\right)(\epsilon / 2) q^{\cdot 2} h(q)+\kappa^{2} f(q)=0,
$$

where $h(q)$ denotes any suitable even function, has been subsequently considered [5]. Equation (4) [describing a second special class] is the object of the present study ${ }^{4}$. This equation has the convenient feature of allowing a discussion of its first integral by rather simple means.

The second term in (4), which governs the energy input and output, is shown as a

${ }^{1}$ Received January 6, 1959; revised manuscript received May 19, 1959. The results presented in this paper were obtained in the course of a study supported by a grant from the National Science Foundation.

${ }^{2}$ Professor of Engineering Mechanics, Stanford University, Stanford, California.

${ }^{3}$ Professor of Mathematics, Ohio State University, Columbus, Ohio.

"Some of the results described here, mostly for the special case $n=1$, can also be found in the recently published book by $H$. Kauderer [6]. 


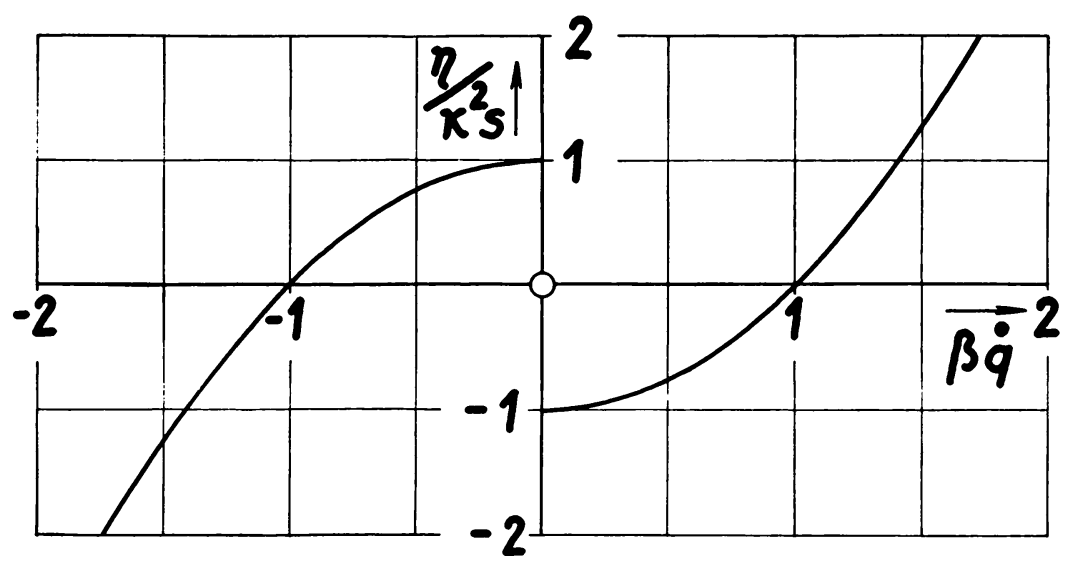

Fra. 1. Second term in Eq. (4)

$\eta=-\left(\kappa^{2} s\right)\left(\operatorname{sgn} q^{*}\right)\left(1-\beta^{2} q^{2}\right)$.

function of $\beta q^{\circ}$ in Fig. 1. In a way familiar from the discussion of the van der Pol Equation (1) it can be shown that the oscillations governed by Eq. (4) also tend towards a stationary regime, a limit cycle. It is the purpose of this paper to study the maximum displacements ("amplitudes") $Q$ of the oscillations governed by Eq. (4) in general and give particular attention to the amplitude $Q^{*}$ of the limit cycle.

2. First integral; amplitude relationship. In Eq. (4), $q$ denotes the dependent variable (displacement), dots indicate derivatives with respect to time; $\kappa^{2}, \beta^{2}, s$ are positive constants. The function $f(q)$, which describes the restoring force, will be subjected to the limitations

$$
\begin{array}{ll}
f(q) \geqq 0 & \text { for } \quad q>0 \\
f(q) \leqq 0 & \text { for } \quad q<0 \\
f(q) \not=0 &
\end{array}
$$

so that only "genuine" restoring forces are admitted. Furthermore, we will start our discussions by requiring $f$ to be an odd function,

$$
f(-q)=-f(q) \text {. }
$$

This restriction, however, is imposed solely for the sake of simplicity of the presentation; it is not essential and will be dropped later (Sec. 4).

Because of the factor $\left(\operatorname{sgn} q^{*}\right)$, there are two different forms of the differential equation, depending on the direction of the motion. However, if we reverse the direction of the coordinate $q$ when the motion changes direction, by putting

$$
\begin{aligned}
& \tilde{q}=q \quad \text { for } \quad q^{\cdot}<0 \\
& \tilde{q}=-q \text { for } q^{\cdot}>0
\end{aligned}
$$

Eq. (4) assumes the single form

$$
\tilde{q}^{\cdot \cdot}+\kappa^{2} s\left[1-\beta^{2}\left(\tilde{q}^{*}\right)^{2}\right]+\kappa^{2} f(\tilde{q})=0,
$$

with $\tilde{q}$ as the dependent variable. 
Writing $U=\tilde{q}^{2} / \kappa^{2}$ we find from (9)

$$
\frac{d U}{d \tilde{q}}+2 s\left(1-\beta^{2} \kappa^{2} U\right)+2 f(\tilde{q})=0,
$$

a first order differential equation for $U$ as a function of $\tilde{q}$. By putting

$$
x=\tilde{q} / s ; \quad y=\frac{U}{s^{2}} ; \quad \lambda=\beta^{2} \kappa^{2} s^{2} ; \quad \frac{f(\tilde{q})}{s}=\phi(x),
$$

we may give differential Equation (10) a non-dimensional form,

$$
\frac{d y}{d x}-2 \lambda y=-2[1+\phi(x)] .
$$

For the initial condition $q=Q_{1}>0$, when $q^{*}=0$, equivalently $x=X_{1}$ for $y=0$, the solution of (12) can be stated as

$$
y=2 e^{2 \lambda x}\left[I\left(X_{1}\right)-I(x)\right]
$$

with $I(x)$ denoting

$$
I(x)=\int_{0}^{x} e^{-2 \lambda \xi}[1+\phi(\xi)] d \xi .
$$

Equation (13a) holds until the motion comes to its next stop $(y=0)$ at $x=X_{2}<0$. Hence

$$
I\left(X_{2}\right)=I\left(X_{1}\right)
$$

provides the relationship between two consecutive maximum displacements $Q_{1}>0$ and $Q_{2}<0$ expressed by their non-dimensional representatives $X_{1}$ and $X_{2}$. We shall refer to (14) as the "amplitude relationship" (taking the liberty of using the term "amplitude" as synonymous with "maximum displacement").

3. Amplitude relationships for special types of restoring forces. We start our discussion by assuming the function $f(q)$ to be proportional to a pure (odd) power $n=$ $2 m+1$ of $q$,

$$
f(q)=\mu^{n-1} q^{n}, \quad(n \text { odd }) .
$$

The function $\phi(x)$, then, turns into

$$
\phi(x)=\gamma_{n} x^{n} \text { with } \quad \gamma_{n}=(\mu s)^{n-1} ;
$$

the expression $I(x)$ in (13b) becomes

$$
I(x)=-\frac{1}{2 \lambda}\left[e^{-2 \lambda x}-1\right]+\gamma_{n} \int_{0}^{x} e^{-2 \lambda \xi} \xi^{n} d \xi .
$$

Carrying out the integration and transferring to the left hand side all terms independent of $x$, we find

$$
\frac{1}{\gamma_{n}}\left[-\frac{(2 \lambda)^{n+1}}{n !} I(x)+\frac{(2 \lambda)^{n}}{n !}+\gamma_{n}\right]=e^{-2 \lambda x}\left[\frac{(2 \lambda)^{n}}{\gamma_{n} n !}+\sum_{n=0}^{n} \frac{(2 \lambda x)^{\nu}}{\nu !}\right] .
$$


Using the symbols $z=2 \lambda x$ and $Z_{i}=2 \lambda X_{i}$ we may abbreviate the right hand side of (18) by $G_{n}(z)$, where

$$
\begin{aligned}
& G_{n}(z)=e^{-z} F_{n}(z), \\
& F_{n}(z)=a_{n}+\sum_{n=1}^{n} \frac{z^{v}}{\nu !},
\end{aligned}
$$

and

$$
a_{n}=1+\frac{(2 \lambda)^{n}}{n ! \gamma_{n}}
$$

Now, the amplitude relationship (14) may be written as

$$
G_{n}\left(Z_{2}\right)=G_{n}\left(Z_{1}\right) \text {. }
$$

Because $F_{n}(z)$, Eq. (20a), is a polynomial of odd degree having real coefficients, $F_{n}(z)$ possesses at least one real zero, $z_{0}$; and, because the coefficients of that polynomial are positive, $z_{0}$ has to be negative. Furthermore we note that

$$
\begin{array}{lll}
G_{n}(z)>0 & \text { for } & z \geqq 0 \\
G_{n}(z) \rightarrow 0 & \text { as } & z \rightarrow \infty \\
G_{n}(z) \rightarrow-\infty & \text { as } & z \rightarrow-\infty .
\end{array}
$$

There exists only one abscissa $z_{1}$ for which $G^{\prime}(z)=0$; it is

$$
z_{1}=-2 \lambda / \gamma_{n}^{1 / n} \text {. }
$$

Since $G_{n}^{\prime \prime}\left(z_{1}\right)<0$ the function $G_{n}(z)$ has a maximum at $z_{1}$. From this and (22) it follows that

$$
z_{0}<z_{1} \text {, or }\left|z_{0}\right|>\left|z_{1}\right| \text {. }
$$

Figure 2 shows a sketch of the general behavior of $G_{n}(z)$.

A special consideration leads to the estimate for $\left|z_{0}\right|$,

$$
\left|z_{0}\right|<\max \left(n, a_{n}\right) \equiv M_{n} \text {. }
$$

The reasoning runs as follows. From Eq. (20a) we obtain for negative arguments $z=-\zeta$ (with $\zeta>0$ )

$$
F(\zeta)=a_{n}-\zeta+\frac{\zeta^{2}}{2 !}\left(1-\frac{\zeta}{3}\right)+\frac{\zeta^{4}}{4 !}\left(1-\frac{\zeta}{5}\right)+\cdots+\frac{\zeta^{n-1}}{(n-1) !}\left(1-\frac{\zeta}{n}\right),
$$

and we want to find a lower bound for the values $\zeta$ that render $F(\zeta)$ negative. If $\zeta=n$, the last term in (24a) vanishes, all other parentheses and, hence, $\zeta$-terms are negative; therefore, for $\zeta \geqq n$ we find $F(\zeta)<0$, provided $a_{n} \leqq n$. But if $a_{n}>n$ then $F(\zeta)<0$ for all $\zeta \geqq a_{n}$.

Hence, we reach the following conclusions in regard to the amplitudes of the oscillation (illustrated by Fig. 2).

(1) The amplitudes $Q_{2}, Q_{3} \ldots$ (being proportional to $X_{j}$ and $Z_{i}$ ) are limited for all (finite odd) values of $n$ in (15) and for any starting value $Q_{1}$. In fact, for any $j \geqq 2$ the inequality 


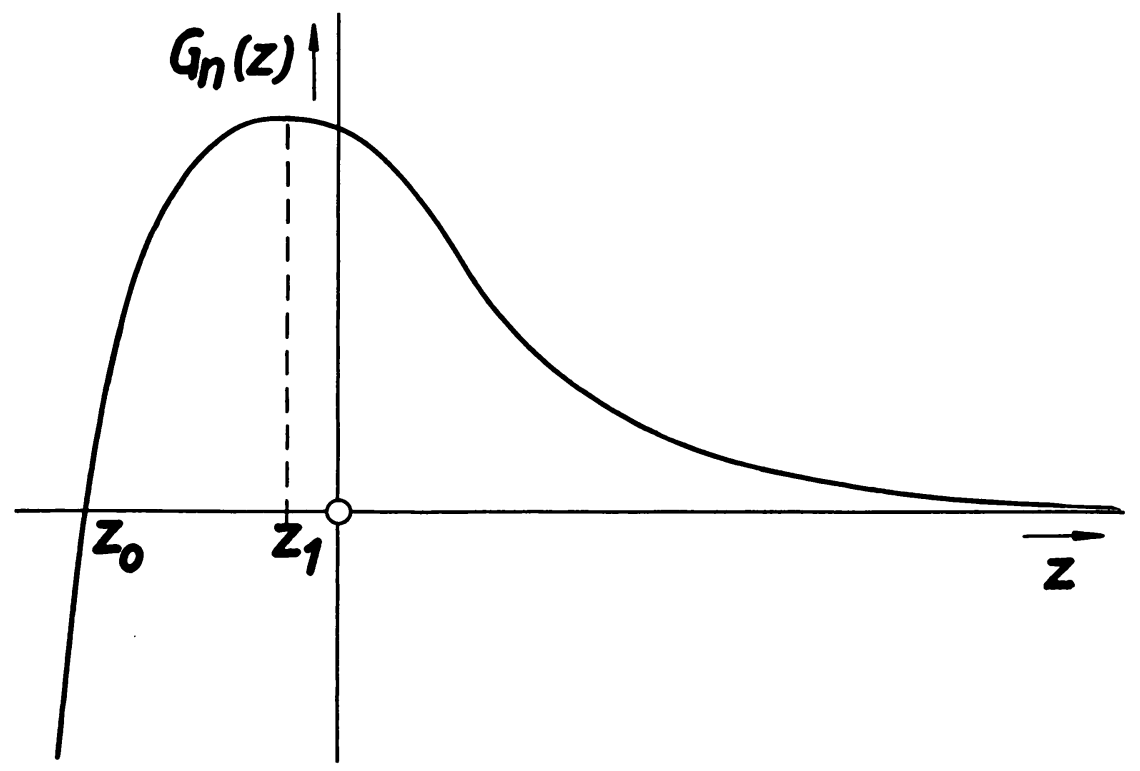

FIG. 2. General behavior of function $G(z)$.

$$
s \gamma_{n}^{-1 / n} \leqq\left|Q_{i}\right| \leqq \frac{s}{2 \lambda} M_{n}
$$

holds, with $M_{n}$ being given by (24).

(2) For two oscillations starting from values $Q_{1}$ and $\bar{Q}_{1}$ respectively, where $Q_{1}>\bar{Q}_{1}>0$ we find

$$
\left|Q_{i}\right|>\left|\bar{Q}_{i}\right| \text { for all } j \geqq 2 .
$$

(3) For any (finite) value of $n$ there exists a uniquely determined limit amplitude $Q^{*}$. The limit amplitude $Q^{*}$ obeys the inequality (25). More specifically, $Q^{*}=(s / 2 \lambda) z^{*}$ is determined by the root $z^{*}$ of the transcendental equation

$$
G_{n}(-z)=G_{n}(z),
$$

which, because of Eqs. (19) and (20), is equivalent to

$$
\tanh z=\left[\sum_{\rho=0}^{(n-1) / 2} \frac{z^{2 \rho+1}}{(2 \rho+1) !}\right] /\left[a_{n}+\sum_{\rho=1}^{(n-1) / 2} \frac{z^{2 \rho}}{(2 \rho) !}\right],
$$

where for $n=1$ the denominator is $a_{1}$.

Equation (28) represents a relationship between $a_{n}$ and $z^{*}$ as a function of $a_{n}$ (i.e. of the parameters of the system); we profitably use (28) for establishing the inverse function $a_{n}\left(z^{*}\right)$. From (28) and (20b) it follows that $z^{*}$ is a monotone increasing function of $\lambda$.

Now, let us consider special values for $n$. The case $n=1$, because of $\gamma_{1}=1$, leads to

$$
G_{1}(z)=e^{-z}\left[a_{1}+z\right]
$$

with

$$
a_{1}=1+2 \lambda \text {. }
$$




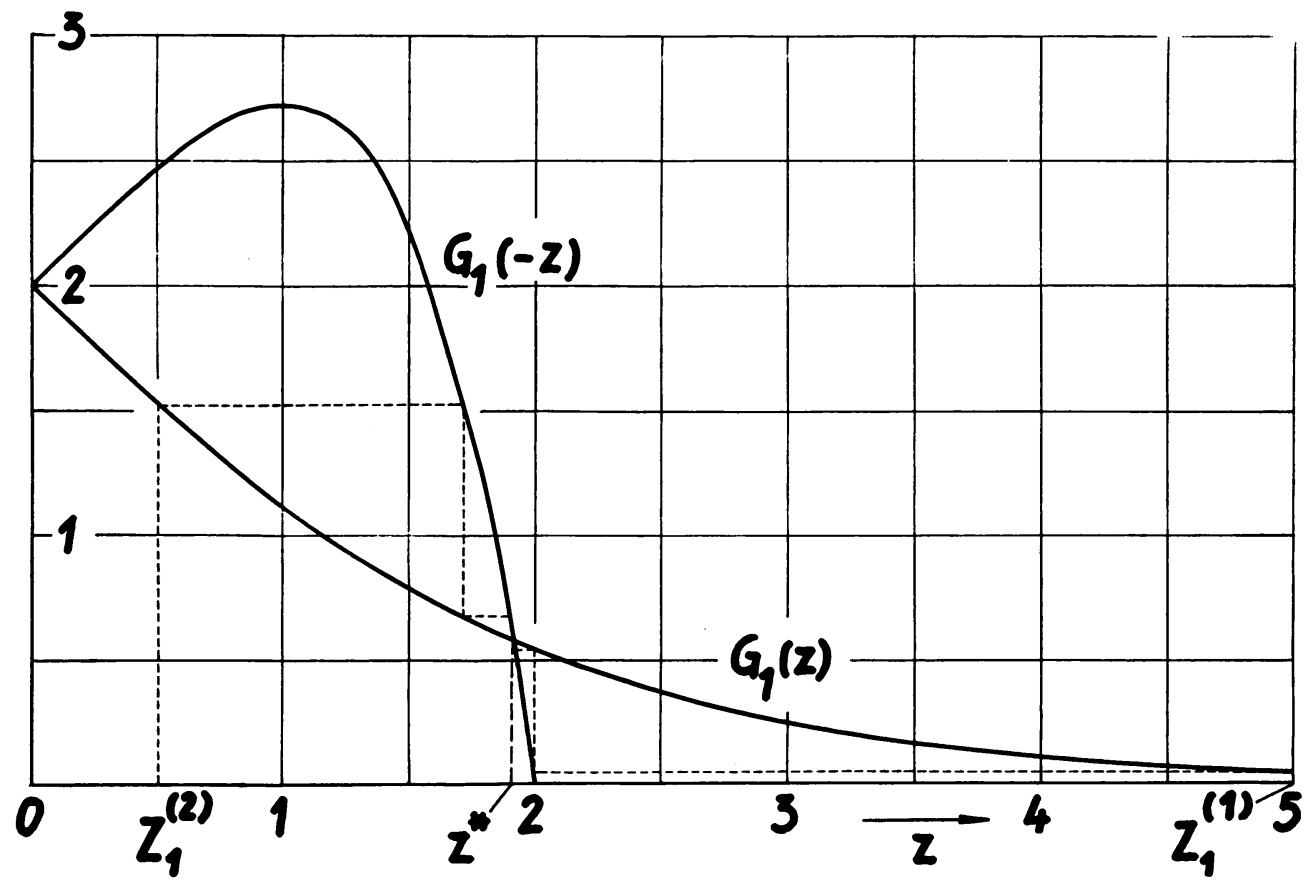

FIg. 3. Functions $G_{1}(z)$ and $G_{1}(-z)$ leading to limit value $z^{*}$.

Figure 3 applies to this case with $\lambda=1 / 2$. From the figure we find the sequences of amplitudes. If $Z_{1}>z^{*}$, the sequence decreases; if $Z_{1}<z^{*}$, the sequence increases to the limit cycle value $z^{*}$. From (28) we obtain

$$
\frac{\tanh z}{z}=\frac{1}{a_{1}}
$$

as the $z^{*}-a_{1}-$ relationship which determines the limit amplitude $z^{*}$. The plot for Eq. (30) is shown in Fig. 4. For the example $2 \lambda=1$ we find

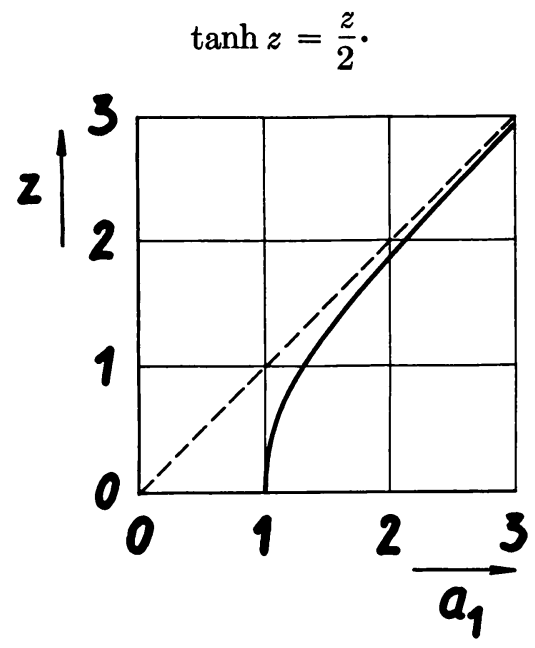

Fig. 4. $a_{1}-z^{*}-$ relationship. 
Since tanh $z \leqq 1$ we have $z^{*}<2$, and the numerical value is $z^{*}=1.915$, equivalently $X^{*}=1.915$ or $Q^{*}=1.915 s$ for the case considered.

Figure 3 is plotted for the parameter value $2 \lambda=1$. The amplitudes of the two sample sequences are

$$
\begin{array}{lll}
Z_{1}^{(1)}=5, & Z_{2}^{(1)}=-1.99, & Z_{3}^{(1)}=1.93 \\
Z_{1}^{(2)}=0.5, & Z_{2}^{(2)}=-1.73, & Z_{3}^{(2)}=1.90 .
\end{array}
$$

For large values of the parameter $2 \lambda$, Eq. (30) may be replaced by

$$
z^{*} \approx a_{1}
$$

from this there follows

$$
X^{*} \approx 1+\frac{1}{2 \lambda} \text { or } Q^{*} \approx s\left(1+\frac{1}{2 \lambda}\right)
$$

Next, we consider the case $n=3$. It leads to

$$
a_{3}=1+\frac{(2 \lambda)^{3}}{\gamma_{3} 3 !}
$$

and to

$$
G_{3}(z)=e^{-z}\left(a_{3}+z+\frac{z^{2}}{2 !}+\frac{z^{3}}{3 !}\right) .
$$

From it the transcendental equation

$$
\tanh z=\frac{6 z+z^{3}}{6 a_{3}+3 z^{2}}
$$

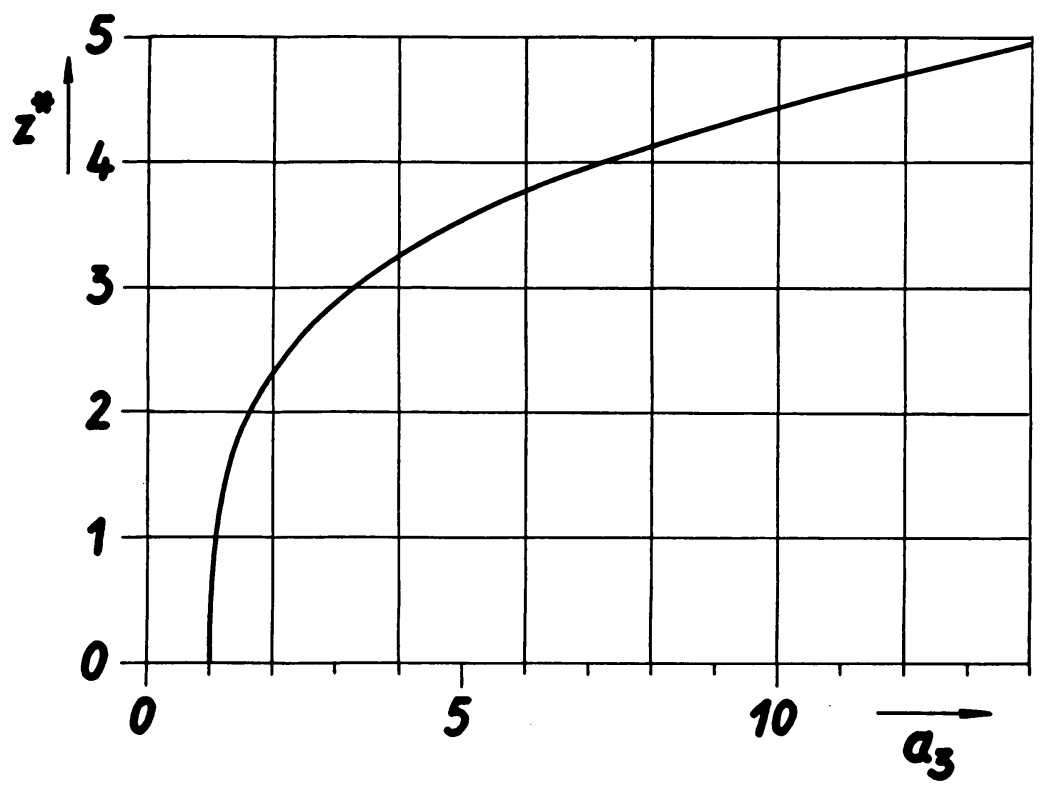

FIG. 5. $a_{3}-z^{*}-$ relationship. 
for the limit value $z^{*}$ is derived. The plot for the $z^{*}-a_{3}$ - relationship is shown in Fig. 5. For $2 \lambda=1, \gamma_{3}=1$, we find $a_{3}=7 / 6$; hence Eq. (34) becomes

$$
\tanh z=\frac{6 z+z^{3}}{7+3 z^{2}} \text {. }
$$

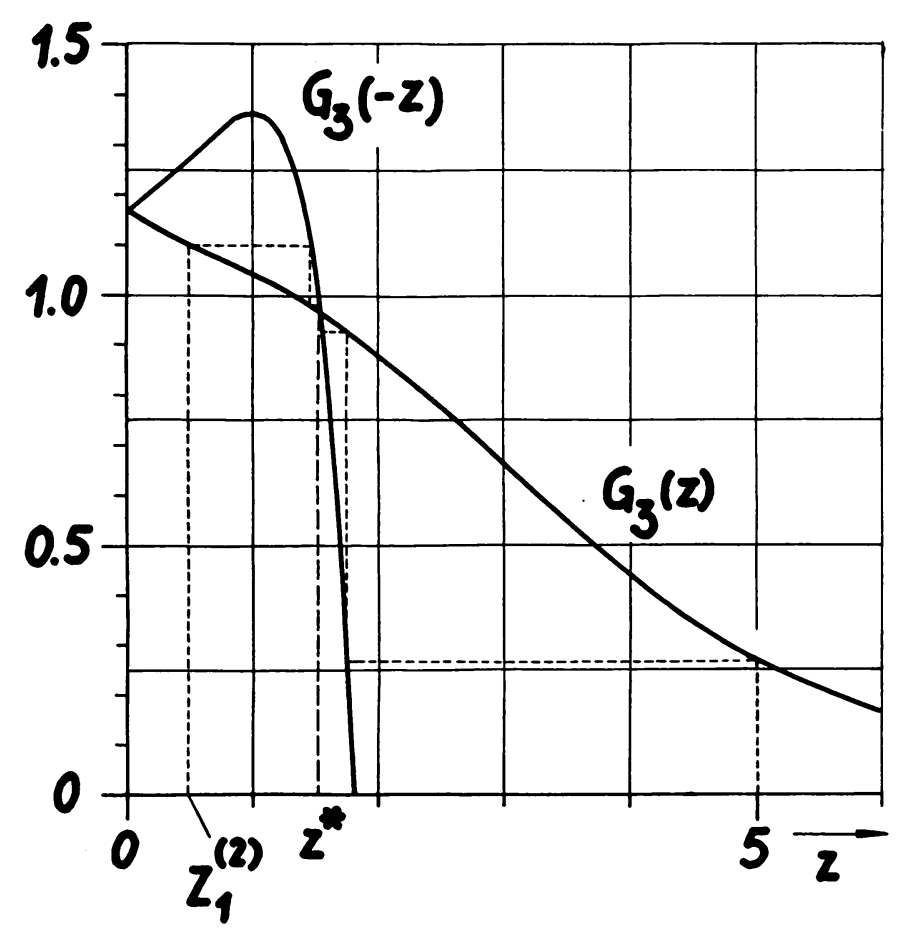

FIG. 6. Functions $G_{3}(z)$ and $G_{3}(-z)$ leading to limit value $z^{*}$.

The plots of $G_{3}(z)$ and $G_{3}(-z)$ are shown in Fig. 6 for the parameter values' $2 \lambda=1$ and $\gamma_{3}=1$. From these plots e.g., the sequences

$$
\begin{array}{lll}
Z_{1}^{(1)}=5, & Z_{2}^{(1)}=-1.76, & Z_{3}^{(1)}=1.55, \\
Z_{1}^{(2)}=0.5, & Z_{2}^{(2)}=-1.46, & Z_{3}^{(2)}=1.52,
\end{array}
$$

for the dimensionless amplitudes can be obtained, both leading to the limit value $z^{*}$, given by the root of Eq. (35). That root is $z^{*}=1.532$, in agreement with the sequences shown above.

Other special cases of odd values $n$ in Eq. (15) can be treated in the same manner.

4. Generalizations. Cases, where $f(q)$ is not a single odd power of $q$, but a polynomial in odd powers, can be treated along the same lines. For instance, if

$$
\begin{gathered}
f(q)=\mu_{1}^{n_{1}-1} q^{n_{1}}+\mu_{2}^{n_{2}-1} q^{n_{2}}, \\
\bar{F}(z)=1+\gamma_{n_{1}} \frac{n_{1} !}{(2 \lambda)^{n_{1}}} \sum_{0}^{n_{1}} \frac{z^{\nu}}{\nu !}+\gamma_{n_{2}} \frac{n_{2} !}{(2 \lambda)^{n_{2}}} \sum_{0}^{n_{2}} \frac{z^{\nu}}{\nu !}
\end{gathered}
$$


Specifically, for $n_{1}=1$ and $n_{2}=3$, because of $\gamma_{1}=1$, Eq. (36) turns into

$$
\vec{F}(z)=1+\frac{1}{2 \lambda}(1+z)+\gamma_{3} \frac{3 !}{(2 \lambda)^{3}}\left(1+z+\frac{z^{2}}{2 !}+\frac{z^{3}}{3 !}\right)
$$

which may be replaced by

$$
\bar{F}(z)=(2 \lambda)^{3}+(2 \lambda)^{2}+6 \gamma_{3}+z\left((2 \lambda)^{2}+6 \gamma_{3}\right)+3 \gamma_{3} z^{2}+\gamma_{3} z^{3} .
$$

For the special values $2 \lambda=1$, and $\gamma_{3}=1$, for instance, the equation (27) reads

$$
\tanh z=\frac{7 z+z^{3}}{8+3 z^{2}} \text {. }
$$

This transcendental equation has the root $z^{*}=1.282$ leading to the limit amplitude $Q^{*}=1.282 s$.

If we drop the restriction, imposed by Eq. (6b), on the function $f(q)$ to be an odd function, and admit non-odd functions $f=f_{0}+f_{0}$ [composed of odd, $f_{0}$, and even, $f_{\text {. }}$, parts] the function $f(\tilde{q})$ in (9) will differ for the swings in the two directions. We find

$$
\begin{aligned}
& f(\tilde{q})=f_{0}(\tilde{q})+f_{e}(\tilde{q}) \text { for } \quad q<0 \\
& f(\tilde{q})=f_{0}(\tilde{q})-f_{0}(\tilde{q}) \text { for } \quad q>0 .
\end{aligned}
$$

Hence, the swings with negative velocities, $q^{*}<0$, and those with positive velocities, $q^{\circ}>0$, will have to be treated separately. The sequences $\left|Z_{2}\right|,\left|Z_{4}\right| \cdots$ will lead to a value $z_{l}^{*}$ developed from expression (40a) in the differential equation; the sequences $\left|Z_{1}\right|,\left|Z_{3}\right| \cdots$ will lead to a value $z_{r}^{*}$ developed from expression (40b) in the differential equation. The limit amplitudes $Q^{*}$ on the left hand side, derived from $z_{l}^{*}$, and those on the right hand side, derived from $z_{r}^{*}$, therefore, in general, will have different absolute values.

\section{REFERENCES}

1. N. Levinson and O. Smith, $A$ general equation for relaxation oscillations, Duke Math. J. 9, 382-403 (1942)

2. See e.g., E. A. Coddington, N. Levinson, Theory of ordinary differential equations, McGraw-Hill, New York, 1955

3. K. Klotter, Free oscillations of systems having quadratic damping and arbitrary restoring forces, J. Appl. Mechanics 22, 493-499 (Dec. 1955)

4. K. Klotter and E. Kreyszig, Über eine besondere Klasse selbsterregter Schwingungen, Ing.-Arch. 25, No. 6, 389-403 (1957)

5. To appear in J. Appl. Mech.

6. H. Kauderer, Nichtlineare Mechanik, Berlin-Göttingen-Heidelberg, 1958, p. 371 et seq. 